\title{
A atuação em Saúde Mental e Atenção Psicossocial em um desastre da mineração em Mariana (MG): um relato de experiência
}

\author{
The performance in Mental Health and Psychosocial Care in a \\ mining disaster in Mariana (MG): an experience report \\ El desempeño en Salud Mental y Atención Psicosocial en un \\ desastre minero en Mariana ( $M G)$ : un relato de experiencia
}

\author{
Marcela Alves de Lima Santos ${ }^{1}$ \\ Maíra Almeida Carvalho ${ }^{2}$ \\ Cláudia Maria Filgueiras Penido ${ }^{3}$
}

\section{Resumo}

SANTOS, M. A. de L.; CARVALHO, M. A.; PENIDO, C. M. F. A atuação em Saúde Mental e Atenção Psicossocial em um desastre da mineração em Mariana (MG): um relato de experiência. Rev. C\&Trópico, v. 45, n. 2, p. 175-186, 2021. DOI: https://doi.org/10.33148/ cetropicov45n2(2021)art11

Este relato de experiência tem como objetivo discutir o contexto e as vivências desenvolvidas no campo da Saúde Mental e Atenção Psicossocial, no município de Mariana (MG), desde o desastre da Samarco/Vale/BHP Billiton, em 2015. Para isso, foram acionadas as memórias do saber construído com trabalhadoras da saúde mental e pesquisadoras no território atingido, em interlocução com atingidos e trabalhadores de saúde. Após os primeiros cuidados psicossociais, foi possível constituir um Plano de Ação envolvendo toda a Rede de Atenção Psicossocial de Mariana que previa, em seu escopo, a criação de uma equipe voltada para as necessidades psicossociais da população atingida. Foram discutidos os desafios dessa construção no Sistema Único de Saúde de forma síntone às especificidades das demandas dessa população vulnerabilizada e o prolongamento do desastre, compreendido como processo em curso no cotidiano de pessoas atingidas e na dinâmica do território. Em conclusão, foi ressaltada a necessidade de práticas alinhadas à dinâmica e realidades das comunidades atingidas, voltadas para a potencialidade dos cuidados construídos no território e em interlocução com o direito à saúde e cidadania.

Palavras-chave: Saúde Mental. Atenção Psicossocial. Desastres. Atenção Primária à Saúde.

1 Doutoranda em Saúde Coletiva (IRR/Fiocruz); Mestre em Saúde Coletiva (IRR/Fiocruz); Terapeuta Ocupacional e Trabalhadora da Rede de Atenção Psicossocial de Mariana (MG).E-mail: marcela_lima5@ hotmail.com. ORCID: https://orcid.org/0000-0002-7579-8067

2 Mestre em Psicologia Social (UFMG); Psicóloga e Trabalhadora da Rede de Atenção Psicossocial de Mariana (MG). E-mail: mairaalmeidac@gmail.com. ORCID: https://orcid.org/0000-0003-2127-9488

3 Professora Adjunta do Departamento de Psicologia e do Programa de Pós-Graduação em Psicologia da Universidade Federal de Minas Gerais (UFMG). E-mail: claudiamfpenido@gmail.com. ORCID: https:// orcid.org/0000-0002-6417-1939 


\section{Abstract}

SANTOS, M. A. de L.; CARVALHO, M. A.; PENIDO, C. M. F. The performance in Mental Health and Psychosocial Care in a mining disaster in Mariana (MG): an experience report. Rev. C\&Trópico, v. 45, n. 2, p. 175-186, 2021. DOI: https://doi.org/10.33148/cetropicov45n2(2021)art11

This experience report aims to discuss the context and activities developed in the field of Mental Health and Psychosocial Care, in the municipality of Mariana (MG), since the mining tailings dam's disaster at Samarco / Vale / BHP Billiton in 2015. For this, memories of the knowledge constructed with mental health workers and researchers in the affected territory were activated and in contact with directly affected population and other health care servers. After the immediate and urgent psychosocial care, it was possible to establish an Action Plan involving the entire Psychosocial Care Network of Mariana, which brought about the creation of a team focused on the psychosocial needs of the population directly affected in its scope. The discussion involved the challenges of this establishment within Brazil's Unified Health System, aligned to the specific demands of this vulnerable population and how the disaster, understood as an ongoing process, continues to influence the daily lives of affected people and the dynamics of the territory. The conclusion showed the need for practices oriented by the dynamics and realities of the affected communities, emphasizing the potential of mental health care built in the territory and in dialogue with the right to health and citizenship.

Keywords: Mental Health. Psychosocial Care. Disasters. Primary Health Care.

\section{Resumen}

SANTOS, M. A. de L.; CARVALHO, M. A.; PENIDO, C. M. F. El desempeño en Salud Mental Atención Psicosocial en un desastre minero en Mariana (MG): un relato de experiencia. Rev. CઐTrópico, v. 45, n. 2, p. 175-186, 2021. DOI: https://doi.org/10.33148/ cetropicov45n2(2021)art11

Este relato de experiencia tiene como objetivo discutir el contexto y las experiencias desarrolladas en el campo de la Salud Mental y la Atención Psicosocial, en el municipio de Mariana (MG), desde el desastre de Samarco / Vale / BHP Billiton en 2015. Para ello, se activaron memorias del conocimiento construido con trabajadores y investigadores de salud mental en el territorio afectado, en diálogo con los afectados y los trabajadores de salud. Luego de la primera atención psicosocial, fue posible crear un Plan de Acción que involucra a toda la Red de Atención Psicosocial de Mariana, que incluyó la creación de un equipo enfocado en las necesidades psicosociales de la población afectada. Los desafíos de esta construcción en el Sistema Único de Salud se discuten en sintonía con las demandas específicas de esta población vulnerable y la extensión del desastre, entendido como un proceso continuo en la vida cotidiana de las personas afectadas y en la dinámica del territorio. En 
conclusión, se destaca la necesidad de prácticas alineadas con las dinámicas y realidades de las comunidades afectadas, orientadas al potencial de atención construido en el territorio y en diálogo con el derecho a la salud y la ciudadanía.

Palabras clave: Salud Mental. Atención Psicosocial. Desastres. Atención Primaria de Salud.

\section{Introdução}

Em 5 de novembro de 2015, ocorreu o rompimento da barragem de rejeitos de Fundão de propriedade da empresa Samarco Mineração S.A. e suas acionistas Vale e BHP Billiton, no município de Mariana, Minas Gerais (MG). Esse desastre da mineração é considerado um dos maiores do mundo, devido à extensão de prejuízos socioambientais. A enxurrada de lama de rejeitos de minério de ferro alterou de forma significativa as vidas de populações e comunidades em todo o percurso que realizou, do distrito de Bento Rodrigues, em Mariana, ao longo da Bacia do Rio Doce, até a cidade de Regência, no Espírito Santo. Na soma dos impactos estão 20 mortes $^{4}$ e danos ambientais imensuráveis, ao longo de 39 municípios atingidos no estado de Minas Gerais e 4 no Espírito Santo.

Em Mariana (MG), o mar de lama destruiu as comunidades de Bento Rodrigues e Paracatu de Baixo, atingindo também, os subdistritos de Paracatu de Cima, Pedras, Campinas, Ponte do Gama e Borba. Nos distritos completamente destruídos, cerca de 350 famílias foram expulsas de seus territórios e sofreram deslocamento forçado da área rural para a sede urbana. Essas populações perderam o seu território de referência e tiveram alterações importantes dos seus modos de vida e de trabalho, bem como a devastação completa das localidades e rompimento dos vínculos sociais e comunitários (IBAMA, 2015).

Na busca de compreender o processo de desterritorialização no contexto de rompimentos de barragens, Santos, Sol e Modena (2020), apontam a importância da relação simbólica das comunidades atingidas com o seu local de origem. A partir da situação de deslocamento forçado, os autores apontam que uma série de alterações são desencadeadas como as de pertencimento e vínculo, além da situação de morte social diante da perda de função constituidora da história de vida das pessoas.

Freitas et al. (2019) em estudo de saúde coletiva sobre os impactos dos desastres em barragens de mineração, à exemplo do desastre da Samarco/Vale/BHP Billiton, identificam que as perdas e rupturas podem provocar danos imediatos para a saúde mental, como também "ampliar e prolongar a medida em que incertezas e inseguranças sobre o futuro se combinam com a ausência de resolutividade dos seus problemas e necessidades pelos órgãos públicos e empresas produtoras dos desastres" (FREITAS, et al, 2019, p.2).

4 Dados oficiais contabilizam 19 mortos no rompimento da Barragem de Fundão da Samarco/Vale/BHP Billiton. Consideramos aqui também um aborto ocorrido na lama, totalizando 20 óbitos. 
Nesse contexto, pensar a condição de saúde mental dos atingidos tornou-se tarefa complexa e que precisa ser discutida e frequentemente analisada. Esse desastre trouxe para a Rede de Atenção Psicossocial (RAPS) de Mariana o desafio de pensar e criar novos modelos assistenciais em saúde mental, bem como a proposição de ações e estratégias inovadoras no contexto da Política Nacional de Saúde Mental, para a garantia do cuidado aos atingidos pela mineração a longo prazo.

Após seis anos, as repercussões psicossociais de um desastre em curso se manifestam em diferentes demandas de uma população que, além das perdas e danos iniciais, tem um cotidiano tomado pela continuidade das violações também nas estratégias institucionais criadas para a reparação de danos. As lutas pela garantia de direitos e a reivindicação pela reparação acontecem em negociações que partem de modelos tecnicistas e burocráticos que vêm se prolongando e causando novas violências.

Entendemos aqui ser fundamental dar visibilidade às potências e desafios na construção de um cuidado em saúde mental longitudinal, a partir dos diferentes impactos e necessidades de saúde que se apresentam. Este trabalho tem como objetivo discutir o contexto e as vivências desenvolvidas no município de Mariana (MG) no campo da Saúde Mental e Atenção Psicossocial desde o desastre da Samarco/Vale/BHP Billiton, considerando os acompanhamentos nessa área ao longo dos últimos seis anos.

Para elaboração deste relato de experiência, foram acionadas as memórias do saber construído como trabalhadoras da saúde mental e pesquisadoras no território atingido. Em interlocução com a escuta das pessoas atingidas e dos trabalhadores que realizam os acompanhamentos a essa população, foram construídas reflexões e saberes sobre os acontecimentos e intervenções nesse campo.

\section{O contexto do território atingido}

Diante do crime-desastre da Samarco/Vale/BHP Billiton e da situação de emergência imposta, foi necessário, inicialmente, redirecionar as ações desenvolvidas pela RAPS municipal prioritariamente para os atendimentos às pessoas atingidas, atuando diretamente nas ações de resposta imediata ao desastre (SANTOS e RIBEIRO, 2017). Naquele momento, foi preciso intervir com o inesperado e abrupto, visto que tal desastre nunca havia sido apresentado como uma hipótese aos trabalhadores de saúde de Mariana. Também não havia no município nenhum plano de contingência que pudesse ofertar amparo ao enfrentamento diante de um acontecimento desta magnitude.

Quando tratamos de um cenário de desastre da mineração, encontramos mais elementos associados a esse contexto de ruptura social, de perda de autonomia e de alteração nos modos de vida. Para além dessas questões, nas interlocuções entre o poder público e as grandes empresas responsáveis pelo desastre existe um cenário de tratativas institucionais que geram novas violações às pessoas atingidas e que atravessam os processos de trabalho e a construção do cuidado em saúde mental e atenção psicossocial.

Noal et al. (2016) discutem a importância de que as respostas em situações críticas sejam organizadas pela gestão pública municipal, estadual e federal, buscando garantir a sustentabilidade e integralidade do processo de cuidado. Dessa forma, 
após a atuação nos primeiros cuidados psicossociais, foi constituído pela Secretaria Municipal de Saúde (SMS), um Plano de Ação envolvendo toda a Rede de Atenção Psicossocial de Mariana,que trazia em seu escopo a criação de uma equipe voltada para as necessidades psicossociais da população atingida (SANTOS e RIBEIRO, 2017).

Conforme apontam Santos, Carvalho e Ribeiro (2019), a equipe construiu ações coletivas em espaços públicos e comunitários da cidade, ações nas escolas, articulações intersetoriais, realizou apoio matricial na Unidade Básica de Saúde (UBS) de Bento/Paracatu e organizou e conduziu discussões em um Fórum Intersetorial de enfrentamento dos conflitos sociais que se aflorou desde a chegada das comunidades rurais à sede urbana.

Entre os desafios enfrentados, podemos citar a construção e desenvolvimento de um trabalho novo em consonância com as diretrizes do Sistema Único de Saúde (SUS), a partir dos princípios da Reforma Sanitária Brasileira e da Reforma Psiquiátrica, e sintonizado às especificidades das demandas dessa população vulnerabilizada. A clareza de que o cuidado em saúde mental deveria ser desenvolvido a partir dos sistemas públicos municipais também exigiu a separação de responsabilidades no que compete à esfera pública nos diferentes níveis de atuação e às empresas do setor privado responsáveis pelo desastre e pelo custeio e financiamento das ações reparatórias. Assim, a construção da atenção psicossocial acompanhou as mudanças constantes nas dinâmicas de reconstrução e os atravessamentos e conflitos de interesses dos diferentes atores envolvidos nos processos.

Segundo Valencio (2014), vários são os efeitos psicossociais do desastre. Muito além das perdas materiais e das necessidades básicas que precisam ser supridas, existem cicatrizes emocionais que afetam a autoestima, a identidade, a sobrevivência e os modos de vida, necessitando de um olhar para as questões de saúde mental individual e coletiva que muitas vezes não são consideradas na retomada das rotinas das populações atingidas. Ao olhar para o curso do desastre, Valencio (2011) também chama atenção para a temporalidade social do sofrimento coletivo, uma vez que o desastre, em sua definição, carrega uma dimensão de sofrimento social que se prolonga num porvir sem perspectiva de uma finalização, extrapolando a perspetiva do tempo cronológico.

Na nossa experiência no campo de pesquisa e de atuação na atenção psicossocial, percebemos impactos para comunidades e populações atingidas em diferentes dimensões. Diante do contato com as famílias nos períodos iniciais, a insistente repetição e atualização das falas sobre o dia do rompimento desvelavam a necessária elaboração de perdas e danos abruptos.

Também era possível perceber que muitas coisas pareciam ter perdido o sentido, como cozinhar sem o fogão à lenha, ou sem a panela de pedra que tanto tinham estima, brincar sem ter o pé de fruta pra subir, alimentar-se sem poder consumir o que era plantado no quintal, ter que percorrer longas distâncias para encontrar os amigos e familiares, estudar em diferentes escolas, estar distante da vizinhança, celebrar as festas tradicionais e encontros religiosos fora do território geográfico e afetivo. Assim, as alterações dos modos de vida e a perda da ruralidade acentuavam cotidianamente o luto diante das perdas materiais e imateriais. 
Ao longo do tempo, as rotinas foram atropeladas por reuniões de negociações assimétricas com as empresas, ou instituições criadas para reparação, trazendo a necessidade de um processo de construção da consciência e aprendizado de uma prática política nas reivindicações por justiça. Além disso, diante dos respingos da lama que continuavam a manchar a rotina dos sujeitos, estes se deparavam com os sucessivos adiamentos dos prazos das entregas dos reassentamentos e descumprimentos de acordos judiciais pelas empresas.

O desastre, compreendido enquanto um processo em curso, continua se constituindo no cotidiano de pessoas atingidas e na dinâmica do território. No momento da escrita deste artigo, a ausência de efetivas reparações, a falta de participação nos planejamentos e projetos de vida e futuro e a angústia e o desamparo pela espera pelas casas e pela reconstrução dos reassentamentos aparecem entre as principais fontes de sofrimento entre a população atingida.

Diante disso, enfatizamos também um cenário de injustiça ambiental, compreendida como consequência de disputas políticas e simbólicas envolvendo os distintos projetos e aplicações dos recursos nos territórios, assim como o uso do poder para impor esses projetos. Essas situações desvelam as condições de pouca participação popular e de assimetrias de poder exercidas por força direta, meios econômicos, políticos e simbólicos em diferentes instâncias (PORTO, PACHECO e LEROY, 2013).

Dessa forma, o trabalho em saúde mental e atenção psicossocial desenvolvidos pelas equipes da RAPS de Mariana, e especialmente pela equipe específica criada para atuação com as populações que sofreram deslocamento forçado, devem estar atentas à realidade social específica que se apresenta, às culturais locais, e à complexidade inerente aos impactos da mineração a uma população atingida por barragem.

\section{O trabalho em Saúde Mental e Atenção Psicossocial}

Ainda que os desastres continuem acontecendo com grande frequência no Brasil, a saúde mental e a atenção psicossocial em contextos de desastres configura-se como um campo de conhecimento e intervenção em construção e pouco desenvolvido ou estruturado no âmbito das políticas públicas brasileiras. Ao longo das últimas duas décadas, diversos esforços têm sido empreendidos para superar essa lacuna e desenvolver técnica e cientificamente este campo.

Pensar a saúde mental e atenção psicossocial em uma situação de desastre exige um conhecimento sobre as políticas públicas e diretrizes nacionais na área e compreensão da rede local onde serão desenvolvidas as ações, dos diferentes atores e da condição de vida em que estão inseridos. A compreensão do desastre e do impacto à vida das comunidades é essencial para uma construção de respostas orientada pelas concepções e diretrizes do SUS. 
O relatório técnico realizado pelo Fórum Mineiro de Saúde Mental sobre os

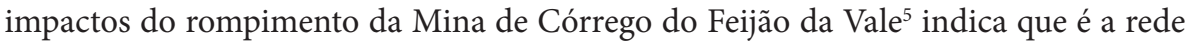
pública de saúde que possui as melhores condições e conhecimentos no campo da saúde mental para atuação nesses contextos. Assim, o SUS é apontado como saída para não se incorrer em violações de direitos humanos, em métodos e práticas psicologizantes e medicalizadoras, respeitando a lógica do território e suas reais necessidades.

Como também aponta o guia elaborado pelo Comitê Permanente Interagências "Diretrizes do IASC sobre Saúde Mental e Apoio Psicossocial em Emergências Humanitárias” (IASC, 2007), as pessoas são afetadas de diferentes formas, demandando diferentes estratégias de apoio. Contudo, a pirâmide de cuidado indicada pelo guia aponta que a maior parte dos sujeitos vão necessitar de cuidados básicos e de segurança, ou de intervenções voltadas para fortalecimento da rede de suporte social. Assim, entendemos que desde os períodos de emergência, uma menor parte da população demandará um suporte em serviços especializados em saúde mental.

Além desses referenciais, é importante citar o manifesto do I Simpósio Internacional de Saúde Mental na Gestão Integral de Riscos e Desastres (2015), com organização da Universidade de Brasília (UnB), do Ministério da Saúde (MS) e da Organização Pan-Americana da Saúde (OPAS). Entre as diretrizes para as ações de Saúde Mental e Atenção Psicossocial são citadas as necessidades: de considerar o princípio da integralidade da atenção de forma intersetorial e transversal, possibilitando a mobilização e participação popular e o controle social; de priorizar questões relativas à saúde numa perspectiva ampliada; da garantia de direitos e do protagonismo dos sujeitos e da comunidade afetada; de uma ampla gama de intervenções de planejamento e de monitoramento de ações e de desenvolver a articulação em redes, a partir das políticas públicas e da valorização do conhecimento e da cultura local.

Diante de um cenário de tantas mudanças e violações de direitos foi preciso ampliar o olhar sobre o sujeito em sua multidimensionalidade e construir propostas de trabalho que fizessem sentido para os atingidos e estimulassem o seu protagonismo. Em Mariana foram executadas ações em saúde mental em emergências e desastres conectadas e contextualizadas à realidade social e construídas em abordagens na própria comunidade, em atuações realizadas junto às equipes de Atenção Primária à Saúde. As ações foram conduzidas em uma perspectiva das coletividades e da compreensão de aspectos do adoecer intrínsecos a esse contexto, a partir de suas referências socioculturais.

Pensando nessas diversas dimensões do sofrimento e nas possibilidades de intervenção no período de reconstrução, durante todo o tempo foi preciso construir ações em saúde de forma contextualizada às vivências das famílias no trabalho, moradia, lazer, estudos, relações sociais, saúde, religião, tanto em seus distritos de origem quanto nesse

5 O relatório técnico sobre os impactos do desastre do rompimento da barragem da mina Córrego do Feijão na saúde mental das populações dos municípios atingidos visa atender à demanda feita pelo Dr. Edmundo Antônio Dias, Procurador Regional Substituto dos Direitos do Cidadão em Minas Gerais, do Ministério Público Federal. Trata-se de solicitação, ao Fórum Mineiro de Saúde Mental, de um estudo técnico junto aos municípios atingidos pelo rompimento da referida barragem, para elaboração de propostas de composição de equipes multiprofissionais e serviços de saúde mental aos municípios atingidos. 
novo contexto. À exemplo, foram construídas estratégias para retomada das atividades cotidianas, os contatos sociais, ou sua potencialização na sede do município de Mariana.

O olhar para as dimensões sociais do adoecer e o enfrentamento a partir das diretrizes da integralidade e da equidade em saúde têm sido fundamentais para a construção de estratégias de saúde voltadas para o fortalecimento e protagonismo das comunidades. Dessa forma, o cuidado tem sido construído em uma perspectiva territorial, humanizada e emancipatória, considerando a demanda pela garantia de direitos e a não ocorrência de novas violações.

Em um contexto marcado pelo deslocamento forçado, é importante ressaltar o processo de vivência de diferentes territórios ao mesmo tempo. Como colocado por Santos (1985), é o uso do território que faz dele objeto de análise social. Assim, é preciso pensar nas relações sociais e diferentes usos feitos pelos grupos populacionais. Diante de uma retirada abrupta, perde-se as referências, estruturas e histórias. Para a população atingida, convive-se com os espaços geográficos destruídos, com aquele em que se vive e com a espera de um reassentamento que tem prazos alterados com frequência. Além disso, é preciso considerar as dimensões simbólicas, afetivas e de poder que caracterizam a apropriação desses diferentes territórios (HAESBAERT, 2019).

Diante dessa realidade, utilizar o território como um espaço de intervenção foi analisado como uma maneira de aproximar do contexto e dos modos de vida dos sujeitos. Essa estratégia foi considerada como central, uma vez que os atingidos precisavam estabelecer novas referências num novo local em que não se reconheciam. Foram construídos espaços terapêuticos fora dos muros e do formato dos dispositivos substitutivos da RAPS, como forma de potencializar as atividades comunitárias, nos espaços das quadras, associações de bairros, praças, escolas e espaços culturais.

Zhouri (2018) aponta que além de um deslocamento físico e material, o sujeito social atingido experimenta um deslocamento social e cultural. Assim, os encontros, sejam nas oficinas, grupos terapêuticos, reuniões ou assembleias, eram considerados espaços potenciais para resgatar a identidade coletiva, possibilitar a elaboração das experiências de perdas, retomar atividades significativas para os sujeitos e também facilitar a construção de redes de apoio e suporte social por meio de encontros entre os moradores.

Entre os aspectos a serem destacados na construção do cuidado em saúde mental, pode-se destacar as práticas interprofissionais dentro das equipes de saúde. A equipe de saúde mental, por exemplo, foi formada por psicólogas, terapeutas ocupacionais, psiquiatra, assistente social, arteterapeuta e músico voltados para um trabalho construído de forma articulada e que transgredisse as barreiras das especialidades. $\mathrm{O}$ foco da construção das intervenções eram os indivíduos e famílias a serem cuidadas e os projetos terapêuticos construídos em equipe - com a participação dos primeiros -, a partir dos diferentes olhares convocados de acordo com o caso.

Além disso, é importante ressaltar a presença da arte e da cultura e práticas integrativas em saúde dentro dessa construção. Reconhecida enquanto uma estratégia importante na construção das ações de respostas em saúde mental, os diferentes recursos terapêuticos nessa área têm se mostrado potentes dentro do trabalho. Nessas 
ações, observamos essa experiência como essencial para a expressão, ressignificação e elaboração do processo de busca de autonomia junto às comunidades atingidas.

Como o trabalho também é construído em rede, quando se tratam de casos de saúde mental graves e persistentes, os serviços de referência são os Centros de Atenção Psicossocial (CAPS) de Mariana. No período do rompimento da barragem, o município contava com dois CAPS, um que atende o público adulto (CAPS I) e outro para atendimento ao público infantojuvenil (CAPSij). Atualmente, além desses dois, foi implantado mais um, voltado para o atendimento de pessoas que fazem uso abusivo de álcool e outras drogas (CAPSad).

Nesses casos, mantém-se a articulação entre os serviços de saúde mental e outros dispositivos da Rede de Proteção que podem ser acionados para a construção do projeto terapêutico. $\mathrm{Na}$ construção desse trabalho intersetorial podemos citar o Conselho Tutelar, os Centro de Referência em Assistência Social (CRAS), os Centro de Referência Especializada em Assistência Social (CREAS) e a Educação, principalmente através das Escolas Municipais de Bento Rodrigues e de Paracatu de Baixo.

Dessa forma, observamos um trabalho desenvolvido de forma territorializada, interprofissional, intersetorial e em articulação com as diferentes demandas dos grupos atingidos. As interlocuções com as comunidades e a leitura crítica das relações que se estabelecem no território embasam um trabalho desenvolvido a partir das diretrizes do SUS e do conceito ampliado de saúde.

\section{Considerações finais}

O trabalho de saúde mental e atenção psicossocial desenvolvido nesse desastre foge aos contextos comumente encontrados nos dispositivos da Rede de Atenção Psicossocial. Além de todas as construções e elaborações subjetivas diante de uma vivência potencialmente traumática, envolve a adaptação a um novo território, a ressignificação de memórias e identidades, a luta por direitos e a construção de novos vínculos e redes de suporte sociais.

A adoção a essa perspectiva de cuidado, portanto, está associada a um duplo movimento de ruptura com uma perspectiva biomédica, focada em patologias. De um lado, evidenciar as práticas de novas violações de direitos realizadas através da patologização e medicalização das manifestações e reações emocionais das populações e comunidades atingidas. De outro, a necessidade de pensar os aspectos do adoecer no contexto de sua realidade e determinação social, ou seja, a partir das condições sócio-históricas e socioeconômicas que produzem e contribuem para a produção de novos desastres.

Diante do contexto encontrado em Mariana, a articulação com a Atenção Primária à Saúde, o trabalho interprofissional e em rede e as construções na perspectiva territorial se apresentam enquanto importantes orientadores dos processos de trabalho. Além disso, há necessidade de que gestores e trabalhadores do SUS estejam atentos aos espaços políticos e de luta pela reparação integral, visto que as respostas institucionais têm sido apontadas como fator de agravamento para a condição de saúde. 
Assim, é importante investir na formação de profissionais para atuação nesse cenário de forma que a atuação no campo possa ser fortalecida e também possibilitar a construção de autonomia junto às comunidades atingidas. Além disso, a produção de mais trabalhos e a divulgação das experiências locais proporcionam maior visibilidade às condições dessas pessoas que continuam vivendo o desastre e lutando pela reparação integral.

Portanto, ao falar de saúde mental e atenção psicossocial, os caminhos das práticas precisam estar alinhados à dinâmica e realidades das comunidades atingidas, voltados para a potencialidade dos cuidados construídos no território e em interlocução com o direito à saúde e cidadania. 


\section{Referências}

FREITAS, C. M. de; BARCELlOS, C.; ASMUS, C. I. R. F.; SIlVA, M. A da.; XAVIER, D. R. Da Samarco em Mariana à Vale em Brumadinho: desastres em barragens de mineração e Saúde Coletiva. Cadernos de Saúde Pública, v. 35, n. 5, 2019. Recuperado de https://www.scielo.br/j/csp/a/5p9ZRBrGkftrmtPBtSLcs9j/?lang=pt. doi:10.1590/0102- 311X00052519

HAESBAERT, R. O mito da desterritorialização: do "fim dos territórios" à multiterritorialidade. $11^{\text {a }}$ ed. Rio de Janeiro: Bertrand Brasil, 2019. p. 396.

INTER-AGENCY STANDING COMMITTEE (IASC). Guidelines on Mental Health and Psychosocial Support in Emergency Settings. 2007. Geneva: Author. Retrieved from: http://www.who.int/mental_health/emergencies/guidelines_iasc_mental_health_psychoso cial.

INSTITUTO BRASILEITO DO MEIO AMBIENTE E DOS RECURSOS HUMANOS RENOVÁVEIS (IBAMA). Laudo técnico preliminar: Impactos ambientais decorrentes do desastre envolvendo o rompimento da barragem de Fundão, em Mariana, Minas Gerais. 2015. Recuperado de http://www.ibama.gov.br/phocadownload/barragemdefundao/laudos/laudo_tecnico_preli minar_Ibama.pdf

MANIFESTO DO I SIMPÓSIO INTERNACIONAL DE SAÚDE MENTAL NA GESTÃO INTEGRAL DE RISCOS E DESASTRES. (2015). Recuperado em 15 de novembro de 2021. Disponível em https://pesquisadesastres.wixsite.com/simposio2015/manifesto.

NOAL,D.S.; VICENTE, L.N.;WEINTRAUB, A.C.A.M.; FAGUNDES, S. M.S.;CABRAL, K. V.; SIMONI, A. C. R.; PACHECO, M. L. L.; PEDROZA, R. L. S.; PULINO, L.H.C.Z.. Estratégia de saúde mental e atenção psicossocial para afetados da Boate Kiss. Psicologia: Ciência e Profissão, v. 36, n. 4, p. 932-945, 2016. doi:10.1590/1982-3703002062016

PORTO, M.F.; PACHECO, T.; LEROY, J. P. (Org.). Injustiça ambiental e saúde no Brasil: o Mapa de Conflitos [online]. Rio de Janeiro: Editora FIOCRUZ, 2013, 306 p. ISBN 978-85-7541-576-4. https://doi.org/10.7476/9788575415764.

SANTOS, M. O retorno do Território. In: SANTOS, Milton et al. (Org.). Território: Globalização e Fragmentação. 4. ed. São Paulo: Hucitec: Anpur, 1998. p. 15-20.

SANTOS, M. A. L., CARVALHO, M. A., \& RIBEIRO, S. R.. O cuidado em saúde mental da população de atingidos na tragédia da Samarco: reflexões a partir da práxis. In: T. M. M. Pinheiro, M. V. Polignano, E. M. A. Goulart, J. de C. Procópio (Orgs.), Mar de lama da Samarco na bacia do rio doce: em busca de respostas. 2019, p. 190-199. Belo Horizonte: Instituto Guaicuy. 
SANTOS, M. A. L., \& RIBEIRO, S. R. . A construção do cuidado psicossocial aos atingidos do desastre de Mariana (MG): Um relato de experiência. In O. S. Filho, \& D. C. Lopes (Orgs.), O psicólogo na redução dos riscos de desastres: teoria e prática, p. 185-196, 2017. São Paulo: Hogrefe CETEPP.

SANTOS, M. A. L., SOL, N. A. A., \& MODENA, C. M. . Território e desterritorialização: o sofrimento social por desastre ambiental decorrente do rompimento de barragens de mineração. Saúde em Debate, v. 44, n. 2, p. 262-271, 2020. Recuperado de https://scielo.br/j/sdeb/a/hNYv4Mw6gvDHBBsvT7vrhMQ/?lang=pt. doi:10.1590/0103-11042020E218

VALENCIO, N. . A sociologia dos desastres: perspectivas para uma sociedade de direitos. In: Conselho Federal de Psicologia, Psicologia de emergências e desastres na América Latina: promoção de direitos e construção de estratégias de atuação. p. 13-30, 2011. Brasília: CFP .

VALENCIO, N. Desastres: tecnicismo e sofrimento social. Ciência \& Saúde Coletiva, v. 19, n. 9, 3631-3644, 2014. Recuperado de https://www.scielo.br/j/csc/a/mgDC4L9wdshN9rJ3GLV3PVv/?lang=pt. doi:10.1590/1413-81232014199.06792014

ZHOURI, A. et al. O desastre no Rio Doce: entre as políticas de reparação e a gestão das afetações. In: Zhouri, Andréa (Org). Mineração: violências e resistências [livro eletrônico]: um campo aberto à produção de conhecimento no Brasil. / 1.ed.- Marabá, PA : Editorial iGuana; ABA, 2018. 711 kb ; e-PUB, p.28-64. 